\title{
On particle acceleration around shocks II: a fully general method for arbitrary shock velocities and scattering media
}

\author{
Pasquale Blasi ${ }^{1}$ and Mario Vietri ${ }^{2}$ \\ ${ }^{1}$ INAF/Osservatorio Astrofisico di Arcetri, Firenze, Italy \\ 2 Scuola Normale Superiore, Pisa, Italy
}

\begin{abstract}
The probability that a particle, crossing the shock along a given direction, be reflected backwards along another direction, was shown to be the key element in determining the spectrum of non-thermal particles accelerated via the Fermi mechanism around a plane-parallel shock in the test-particle limit. Here an explicit equation for this probability distribution is given, for both the upstream and downstream sections. Though analytically intractable, this equation is solved numerically, allowing the determination of the spectrum in full generality, without limitation to shock speed or scattering properties. A number of cases is then computed, making contact with previous numerical work, in all regimes: Newtonian, trans-relativistic, and fully relativistic.
\end{abstract}

Subject headings: shock waves - cosmic rays

\section{Introduction}

It is possible to formulate particle acceleration around shocks, in the test particle limit, in an exact form, for arbitrary shock speeds, and arbitrary anisotropies in the particle distribution function (Paper I, Vietri 2003). It turns out that the physically relevant boundary conditions are those at the shock, not those at downstream infinity. These conditions at the shock fix the particle spectrum which was shown to be (under some simplifying assumptions) an exact power-law in the particle momentum, independent of the shock speed and of all assumptions about the scattering properties of the medium.

The exact value of the spectral index depends in a detailed way upon the diffusion properties of the media involved. It was shown in Paper I that this dependence is encapsulated in two functions, $P_{u}\left(\mu_{\circ}, \mu\right)$ and $P_{d}\left(\mu_{\circ}, \mu\right)$ respectively, which denote the conditional probabilities that a particle entering the upstream (downstream) medium along a direction $\mu_{\circ}$, 
will leave it for the downstream (upstream) section along a direction $\mu^{1}$. In paper I (but see also Freiling, Vietri and Yurko 2003 for some tricky mathematical details), these functions were built from the eigenfunctions of the angular part of the scattering problem. Though conceptually satisfactory, this procedure is however computationally tricky, and one may wonder whether, given the scattering law for the particles, it may be possible to determine an equation for the scattering probabilities that yields them directly, without having to build the infinitely many eigenfunctions of the angular equation. It turns out that this is actually possible, by drawing upon the analogy with scattering atmospheres (Chandrasekhar 1949), and by using an obvious symmetry property of the problem at hand.

It is the purpose of this paper to present this derivation, and a handful of illustrative computations, with the major aim of showing the ability of the method to reproduce wellestablished results, drawn from the literature. A more detailed analysis of the spectra in several interesting cases, including the important hyperrelativistic regime, will be presented elsewhere.

The plan of the paper is as follows: in section 2, we present the derivation of the equations which determine $P_{d}\left(\mu_{\circ}, \mu\right)$ and $P_{u}\left(\mu_{\circ}, \mu\right)$. In section 3 , we show that a diffusive equation identical to that originally given by Kirk and Schneider (1987) is recovered in the Fokker-Planck regime (or small-pitch-angle scattering, SPAS) of our scattering equation, Eq. 2 . In section 4, we describe our full method to compute numerically the spectral slope and angular distribution function. In section 5 we show that our method reproduces very well results which have already appeared in the literature, in the newtonian, trans-relativistic and fully relativistic regimes (the ultra-relativistic regime will be discussed in forthcoming publication). We conclude in section 6 .

\section{The equations for $P_{d}$ and $P_{u}$}

In paper I, we showed that a suitable, relativistically covariant (though not manifestly so) equation for the transport of the particle distribution function for arbitrary pitch angle scattering in the presence of a large-coherence length magnetic field is:

$$
\gamma(u+\mu) \frac{\partial f}{\partial z}=\int\left(-w_{e}\left(\mu^{\prime}, \mu, \phi, \phi^{\prime}\right) f(\mu, \phi)+w_{e}\left(\mu, \mu^{\prime}, \phi, \phi^{\prime}\right) f\left(\mu^{\prime}, \phi^{\prime}\right)\right) d \mu^{\prime} d \phi^{\prime}+\omega \frac{\partial f}{\partial \phi} .
$$

In this equation, $z$ is the distance from the shock along the shock normal, in the shock frame. All other quantities are measured in the fluid frame: $u$ is the fluid speed with respect to

\footnotetext{
${ }^{1}$ In these expressions, all angles are measured in the fluid frame.
} 
the shock, in units of $c$, and $\gamma=1 / \sqrt{1-u^{2}}$ its associated Lorentz factor, $f$ is the DF of particles with impulse $p$ in the fluid frame (which, by assumption, does not change because we assumed pitch-angle scattering), and $w_{e}\left(\mu, \mu^{\prime}, \phi, \phi^{\prime}\right)$ is the scattering probability that a particle, originally moving along a direction making an angle with the shock normal such that its cosine is $\mu^{\prime}$, and along $\phi^{\prime}$, be deflected along a new direction $\mu, \phi$. The fluid is assumed to move in the positive $z$ direction, so that its speed is at all places $>0$, and the angles are measured from the direction of motion of the fluid.

The scattering is assumed linear (thus these results apply only in the test particle approximation), but the scattering angle is nowhere assumed small: we shall derive results for the diffusive approximation in a later section. This equation is the plane-geometry, fully relativistic version of an equation originally developed by Gleeson and Axford (1967) for spherically symmetric, non-relativistic shocks.

Compared with Eq. 7 of Paper I, the injection has been dropped because we are considering particles at very large energies, much larger than the injection energy; we now also specialize to the case in which no long-coherence length magnetic field is present. The treatment of the problem in full generality will be presented elsewhere (Morlino, Blasi and Vietri, in preparation).

Besides dropping the term $\omega(\partial f / \partial \phi)$, we remark that the system has now become symmetric for rotations around the shock normal, so that the DF $f$ will be independent of the angle $\phi$. Furthermore, the elemental scattering probability $w_{e}$ can depend only on the angle $\phi-\phi^{\prime}$ between the incoming and outgoing direction, $w_{e}=w_{e}\left(\mu, \mu^{\prime}, \phi-\phi^{\prime}\right)$, even though it can depend separately upon $\mu$ and $\mu^{\prime}$. In this symmetric case, we can average the previous equation over either $\phi$ or $\phi^{\prime}$, obtaining

$$
\gamma(u+\mu) \frac{\partial f}{\partial z}=\int\left(-w\left(\mu^{\prime}, \mu\right) f(\mu)+w\left(\mu, \mu^{\prime}\right) f\left(\mu^{\prime}\right)\right) d \mu^{\prime} d \phi^{\prime}
$$

where

$$
w\left(\mu, \mu^{\prime}\right) \equiv \frac{1}{2 \pi} \int w_{e}\left(\mu, \mu^{\prime}, \phi-\phi^{\prime}\right) d \phi,
$$

an equation which will be used later on.

\subsection{The downstream case}

In order to fix the ideas, we consider the problem of determining $P_{d}$, the conditional probability for the downstream frame; we shall explain later how to adapt these results to the upstream frame. Take the origin of coordinates to be located at the shock, the downstream 
section to be at $z>0$, and define $u$ as the modulus of the shock speed with respect to the fluid. We are using variables in the fluid frame: $\mu$ is thus the cosine of the angle between the particle speed and the shock normal, in the fluid frame. This means that the particles which can cross from upstream to downstream (i.e., those with a positive component along the shock normal of the velocity with respect to the shock) are those with

$$
u+\mu>0
$$

while those that cross the shock backwards, from the downstream to the upstream section, have

$$
u+\mu<0
$$

Now let $f$ represent a beam of particles, all entering the downstream section along the same direction, $\mu_{\circ}$; we showed in paper I that the particle flux across a surface fixed in the shock frame, but expressed in downstream coordinates, is given by:

$$
F \equiv F_{\circ} \gamma=\int \gamma(u+\mu) f d \mu
$$

We consider now a pencil beam of particles, all moving initially in the direction $\mu_{\circ}$. Thus, at the shock:

$$
f(\mu)=\frac{F_{\circ}}{u+\mu} \delta\left(\mu-\mu_{\circ}\right) \quad u+\mu \geq 0,
$$

with $F_{\circ}$ an obvious normalization. It was shown in paper I that Eq. 7 provides a suitable boundary condition for Eq. 2, which then returns the outgoing flux; by definition, this is precisely the required $P_{d}$. We thus have

$$
-\left(u_{d}+\mu\right) f(\mu)=F_{\circ} P_{d}\left(\mu_{\circ}, \mu\right) \quad u+\mu<0 .
$$

To begin, let us define the inward $\left(\left.f_{+} \equiv f(\mu)\right|_{u+\mu>0}\right)$ and outward $\left(\left.f_{-} \equiv f(\mu)\right|_{u+\mu<0}\right)$ parts of the distribution function, and let us consider a surface at a distance $z$ from the shock, fixed in the shock frame. The flux of particles moving backwards toward the shock, through this surface, is:

$$
\gamma(u+\mu) f_{-}(\mu)=-\int_{-u}^{1} f_{+}\left(\mu^{\prime}\right) \gamma\left(u+\mu^{\prime}\right) P_{d}\left(\mu^{\prime}, \mu\right) d \mu^{\prime} .
$$

The minus sign accounts for the fact that $u+\mu<0$, while all terms inside the integral are positive.

Strictly speaking, in the above equation, we should have written $P_{d}\left(\mu^{\prime}, \mu, z\right)$ instead of simply $P_{d}\left(\mu^{\prime}, \mu\right)$. In other words, for a general situation, we cannot assume that the conditional probability $P_{d}$ be independent of its location inside the downstream region. However, 
in this case this assumption is fully justified, because the downstream region is semi-infinite: in other words, it remains identical to itself whenever we add or subtract finite regions, and its diffusive properties also remain unaltered whenever we add or subtract finite regions. It follows that the conditional probability $P_{d}$ from some finite $z$ to downstream infinity must be exactly identical to that from 0 to downstream infinity. This invariance principle, and this whole discussion, are identical to those in Chandrasekhar (1949, Chapter 4, Sections 28-29), and provide an exact justification for the equation above.

We now differentiate the above with respect to $z$ :

$$
\gamma(u+\mu) \frac{\partial f_{-}}{\partial z}=-\int_{-u}^{1} d \mu^{\prime} \gamma\left(u+\mu^{\prime}\right) \frac{\partial f_{+}}{\partial z} P_{d}\left(\mu^{\prime}, \mu\right)
$$

while Eq. 2 gives us

$$
\begin{gathered}
\gamma(u+\mu) \frac{\partial f_{-}}{\partial z}=-d(\mu) f_{-}+\int_{-u}^{1} d \mu^{\prime} w\left(\mu, \mu^{\prime}\right) f_{+}\left(\mu^{\prime}\right)+\int_{-1}^{-u} d \mu^{\prime} w\left(\mu, \mu^{\prime}\right) f_{-}\left(\mu^{\prime}\right) \\
\gamma(u+\mu) \frac{\partial f_{+}}{\partial z}=-d(\mu) f_{+}+\int_{-u}^{1} d \mu^{\prime} w\left(\mu, \mu^{\prime}\right) f_{+}\left(\mu^{\prime}\right)+\int_{-1}^{-u} d \mu^{\prime} w\left(\mu, \mu^{\prime}\right) f_{-}\left(\mu^{\prime}\right),
\end{gathered}
$$

where we used the shorthand

$$
d(\mu) \equiv \int w\left(\mu^{\prime}, \mu\right) d \mu^{\prime} .
$$

We now evaluate Eq. 11 at $z=0$, using Eqs. 7 and 8, to obtain

$$
\left.\gamma(u+\mu) \frac{\partial f_{-}}{\partial z}\right|_{\circ}=\frac{F_{\circ} d(\mu)}{\gamma(u+\mu)} P_{d}\left(\mu_{\circ}, \mu\right)+\frac{F_{\circ} w\left(\mu, \mu_{\circ}\right)}{\gamma\left(u+\mu_{\circ}\right)}-\int_{-1}^{-u} d \mu^{\prime} \frac{w\left(\mu, \mu^{\prime}\right) F_{\circ} P_{d}\left(\mu_{\circ}, \mu^{\prime}\right)}{\gamma\left(u+\mu^{\prime}\right)} .
$$

We can now proceed analogously for Eq. 12: we again evaluate it at $z=0$, and use Eqs. 7 and 8, to obtain:

$$
\left.\gamma(u+\mu) \frac{\partial f_{+}}{\partial z}\right|_{\circ}=-\frac{F_{\circ} d(\mu)}{\gamma(u+\mu)} \delta\left(\mu-\mu_{\circ}\right)+\frac{F_{\circ} w\left(\mu, \mu_{\circ}\right)}{\gamma\left(u+\mu_{\circ}\right)}-\int_{-1}^{-u} d \mu^{\prime} \frac{w\left(\mu, \mu^{\prime}\right) F_{\circ} P_{d}\left(\mu_{\circ}, \mu^{\prime}\right)}{\gamma\left(u+\mu^{\prime}\right)} .
$$

As our last step, we plug Eqs. 14 and 15 into Eq. 10 to obtain:

$$
\begin{gathered}
P_{d}\left(\mu_{\circ}, \mu\right)\left(\frac{d\left(\mu_{\circ}\right)}{u+\mu_{\circ}}-\frac{d(\mu)}{u+\mu}\right)=\frac{w\left(\mu, \mu_{\circ}\right)}{u+\mu_{\circ}}+\int_{-u}^{1} d \mu^{\prime} \frac{P_{d}\left(\mu^{\prime}, \mu\right) w\left(\mu^{\prime}, \mu_{\circ}\right)}{u+\mu_{\circ}}- \\
\int_{-1}^{-u} d \mu^{\prime} \frac{P_{d}\left(\mu_{\circ}, \mu^{\prime}\right) w\left(\mu, \mu^{\prime}\right)}{u+\mu^{\prime}}-\int_{-u}^{1} d \mu^{\prime} P_{d}\left(\mu^{\prime}, \mu\right) \int_{-1}^{-u} d \mu^{\prime \prime} \frac{w\left(\mu^{\prime}, \mu^{\prime \prime}\right) P_{d}\left(\mu_{\circ}, \mu^{\prime \prime}\right)}{u+\mu^{\prime \prime}}
\end{gathered}
$$

which is the sought-after result: an equation relating $P_{d}$ only to the diffusion probability $w$. 


\subsection{The upstream case}

A completely analogous derivation holds in this case. The pencil beam is now entering the upstream section, so that, at the shock, we have:

$$
f(\mu)=-\frac{F_{\circ}}{u+\mu} \delta\left(\mu-\mu_{\circ}\right) \quad u+\mu \leq 0
$$

and we have the obvious definition, from paper I:

$$
f(\mu)=\frac{F_{\circ}}{u+\mu} P_{u}\left(\mu_{\circ}, \mu\right) \quad u+\mu>0 .
$$

At a distance $z$ from the shock the flux of particles moving toward the shock is

$$
\gamma(u+\mu) \frac{\partial f_{+}}{\partial z}=-\int_{-1}^{-u} d \mu^{\prime} \gamma\left(u+\mu^{\prime}\right) P_{u}\left(\mu^{\prime}, \mu\right) \frac{\partial f_{-}}{\partial z}
$$

where the same argument about the use of $P_{u}\left(\mu^{\prime}, \mu\right)$ instead of $P_{u}\left(\mu^{\prime}, \mu, z\right)$ applies as above. We may evaluate the above equation at $z=0$ using Eqs. 11 and 12 (which still hold), and Eqs. 17 and 18, and plug the result into Eq. 19, to obtain

$$
\begin{gathered}
P_{u}\left(\mu_{\circ}, \mu\right)\left(\frac{d\left(\mu_{\circ}\right)}{u+\mu_{\circ}}-\frac{d(\mu)}{u+\mu}\right)=\frac{w\left(\mu, \mu_{\circ}\right)}{u+\mu_{\circ}}-\int_{-u}^{1} d \mu^{\prime} \frac{w\left(\mu, \mu^{\prime}\right) P_{u}\left(\mu_{\circ}, \mu^{\prime}\right)}{u+\mu^{\prime}}+ \\
\int_{-1}^{-u} d \mu^{\prime} \frac{w\left(\mu^{\prime}, \mu_{\circ}\right) P_{u}\left(\mu^{\prime}, \mu\right)}{u+\mu_{\circ}}-\int_{-1}^{-u} d \mu^{\prime} P_{u}\left(\mu^{\prime}, \mu\right) \int_{-u}^{1} d \mu^{\prime \prime} \frac{w\left(\mu^{\prime}, \mu^{\prime \prime}\right) P_{u}\left(\mu_{\circ}, \mu^{\prime \prime}\right)}{u+\mu^{\prime \prime}}
\end{gathered}
$$

which is our other final result.

\section{The small-pitch-angle limit}

In this section, we wish to derive the small pitch-angle scattering (SPAS) limit of our scattering equation, which holds for arbitrary deflection angles, not just small ones. Before proceeding with the derivation of the Fokker-Planck equation, we need to establish a very useful result. The function $w$ is not completely arbitrary, but is subject to the following requirement. We expect that, when the distribution function (DF) is isotropic (i.e., when $f(\mu)=$ const.), there will be no further evolution: in other words, we expect in this case the RHS of the above equation to vanish. For this to occur, we shall not take the obvious condition

$$
\int_{-1}^{+1} w\left(\mu, \mu^{\prime}\right) d \mu^{\prime}=\int_{-1}^{+1} w\left(\mu^{\prime}, \mu\right) d \mu^{\prime}
$$


but its stronger version,

$$
w\left(\mu, \mu^{\prime}\right)=w\left(\mu^{\prime}, \mu\right) .
$$

The above equation is a statement of detailed balance, which we may assume to hold because these scattering processes are exactly those which lead to isotropization of the DF, if thermal equilibrium were to hold. In our case we have neglected (for excellent, well-known reasons) the exchange of energy of the particles with the background fluid, so we cannot expect true thermal equilibrium to hold, but since we have included all major scattering processes, we expect equilibrium (i.e., detailed balance except for energy equipartition) to hold at least in so far as the angular part is concerned. It is probably worthwhile to remark that a similar assumption on $w$ is made by Chandrasekhar (1949, Ch. IV, Section 31, Eq. 29-1).

We now rewrite Eq. 2 by means of a new scattering probability, $W\left(\mu^{\prime} ; \alpha\right)$, to be defined as follows:

$$
W\left(\mu^{\prime} ; \alpha\right) d \alpha \equiv w\left(\mu^{\prime}+\alpha, \mu^{\prime}\right) d\left(\mu^{\prime}+\alpha\right) .
$$

$W$ is simply the probability that a particle is deflected from its initial direction along $\mu^{\prime}$, to a new direction $\mu^{\prime}+\alpha$. By means of this new quantity Eq. 2 can be recast as:

$$
\gamma(u+\mu) \frac{\partial f}{\partial z}=\int_{-1}^{+1} d \alpha(W(\mu-\alpha ; \alpha) f(\mu-\alpha)-W(\mu ;-\alpha) f(\mu))
$$

while the detailed balance equation becomes:

$$
W(\mu-\alpha ; \alpha)=W(\mu ;-\alpha) .
$$

The scattering equation in the form 24 is exactly identical to that given by Landau and Lifshtiz (1984, Section 21, Eq. 21.1), so that their derivation of the Fokker-Planck immediately applies. Please note however that our sign convention differs from theirs: what we call $\alpha$ is for them $-q$. Following step by step their derivation we find that

$$
\gamma(u+\mu) \frac{\partial f}{\partial z} \approx \frac{\partial}{\partial \mu}\left(A f+B \frac{\partial f}{\partial \mu}\right)
$$

with the definitions

$$
\begin{gathered}
A \equiv-\int_{-\infty}^{+\infty} d \alpha \alpha W(\mu ; \alpha)+\frac{\partial B}{\partial \mu} \\
B \equiv \frac{1}{2} \int_{-\infty}^{+\infty} d \alpha \alpha^{2} W(\mu ; \alpha) .
\end{gathered}
$$

In the two integrals above, we have taken the integration range to extend from $-\infty$ to $+\infty$, because we have assumed the validity of the SPAS regime, in which case $W(\mu ; \alpha)$ has one very strong, narrow peak around $\alpha=0$ : 
We show now that, as a consequence of detailed balance, $A=0$. We first notice that

$$
\frac{\partial}{\partial \mu} W(\mu ; \alpha)=\frac{\partial}{\partial \mu} W\left(\mu^{\prime} ; \mu-\mu^{\prime}\right)
$$

where $\mu^{\prime}=\mu+\alpha$, because of detailed balance, so that

$$
\frac{\partial}{\partial \mu} W(\mu ; \alpha)=\frac{\partial}{\partial \alpha} W\left(\mu^{\prime} ; \alpha\right) .
$$

We use the above into

$$
\frac{\partial B}{\partial \mu}=\frac{1}{2} \int_{-\infty}^{+\infty} d \alpha \alpha^{2} \frac{\partial}{\partial \mu} W(\mu ; \alpha)=\frac{1}{2} \int_{-\infty}^{+\infty} d \alpha \alpha^{2} \frac{\partial}{\partial \alpha} W\left(\mu^{\prime} ; \alpha\right)
$$

which can now be integrated by parts to yield

$$
\frac{\partial B}{\partial \mu}=-\int_{-\infty}^{+\infty} d \alpha \alpha W\left(\mu^{\prime} ; \alpha\right) .
$$

We now use again detailed balance, and the variable change $x \equiv-\alpha$ in the definite integral above, to obtain

$$
\frac{\partial B}{\partial \mu}=\int_{-\infty}^{+\infty} d \alpha \alpha W(\mu ; \alpha)
$$

which, when inserted into Eq. 27, yields $A=0$. We thus obtain, for the scattering equation in the SPAS, or Fokker-Planck, limit:

$$
\gamma(u+\mu) \frac{\partial f}{\partial z}=\frac{\partial}{\partial \mu}\left(B \frac{\partial f}{\partial \mu}\right),
$$

with $B$ given by Eq. 28. The use of detailed balance implies that the scattering integral reduces, in the SPAS limit, to the divergence of a vector proportional to the gradient of the $\mathrm{DF}$, exactly like in the classical heat conduction problem.

As a simple test of this treatment, we now re-derive a well-known result, i.e., that, in the isotropic limit,

$$
B=K\left(1-\mu^{2}\right)
$$

with $K$ a constant. The isotropic limit means that the scattering coefficient probability $W$ can only depend on the angle $\theta-\theta^{\prime}$ between the directions of motion. We shall take thus

$$
W(\mu ; \alpha)=N f(\xi)
$$

where

$$
\xi \equiv \frac{\sin \left(\theta-\theta^{\prime}\right)}{\sigma}
$$


with $\sigma$ a parameter which we take $\sigma \ll 1$, in order to enforce the SPAS regime. Also, we assume that $f(x)$ is an even function of $x$, with a single minimum around $x=0$ (again, SPAS regime); we also assume, for the moment, that $W$ is normalized to unity:

$$
\int_{-\infty}^{+\infty} d \alpha N f(\alpha)=1
$$

and will come back to this assumption in a moment.

We now compute $B$ from Eq. 28. Since we shall be taking the limit $\sigma \rightarrow 0$, only the argument $\sin \left(\theta-\theta^{\prime}\right) \approx \sigma \ll 1$ will contribute to the integral, so that, defining $\epsilon \equiv \theta-\theta^{\prime}$, we find

$$
\begin{aligned}
\xi & \approx \frac{\epsilon}{\sigma} \\
\alpha \equiv \mu^{\prime}-\mu=\cos \theta^{\prime}-\cos \theta & =\cos (\theta+\epsilon)-\cos \theta \approx-\sin \theta \epsilon
\end{aligned}
$$

so that the normalization condition, Eq. 38 requires

$$
N=\left(\sin \theta \sigma \int_{-\infty}^{+\infty} f\left(\frac{\epsilon}{\sigma}\right) d \frac{\epsilon}{\sigma}\right)^{-1} .
$$

$B$ then becomes

$$
\begin{array}{r}
B=\frac{1}{2} \int_{-\infty}^{+\infty} d \alpha \alpha^{2} W(\mu ; \alpha) \approx \frac{1}{2} N \sigma^{3} \sin ^{3} \theta \int_{-\infty}^{+\infty} f(\xi) \xi^{2} d \xi= \\
\sin ^{2} \theta \sigma^{2} \frac{\int_{-\infty}^{+\infty} \xi^{2} f(\xi) d \xi}{\int_{-\infty}^{+\infty} f(x) d x}
\end{array}
$$

which is clearly identical to Eq. 35. If we now assume $W$ to have arbitrary normalization (because it represents the probability of scattering per unit time), we see that the above derivation is changed only through an inessential multiplicative constant. This completes our derivation.

\subsection{Scattering probability}

Later on, we shall compare numerical results based upon our new approach with those which previous authors have obtained in the fully isotropic, small pitch angle scattering limit. In order to do this, we have to determine a suitable form for $w$ in this case. It is clear that any function very strongly peaked around the forward direction simulates the SPAS regime, like, for instance,

$$
w \propto e^{-\left(\mu-\mu^{\prime}\right)^{2} / 2 \sigma^{2}}, \quad \sigma \ll 1
$$


However, not all such peaked functions reproduce the isotropic case, which is defined as that in which the elemental scattering probability $w_{e}$ is independent of anything except for the deflection angle:

$$
w_{e}\left(\mu, \mu^{\prime}, \phi, \phi^{\prime}\right)=w(\cos \Theta)
$$

with $\Theta$ given by

$$
\cos \Theta \equiv \mu \mu^{\prime}+\sqrt{1-\mu^{2}} \sqrt{1-\mu^{\prime 2}} \cos \left(\phi-\phi^{\prime}\right) .
$$

We have seen above that the transport equation in the SPAS regime does not depend upon the exact form of the scattering function $w_{e}$, so that we are free to choose any analytically tractable one. We choose:

$$
w_{e}(\cos \Theta)=\frac{1}{\sigma} e^{-\frac{1-\cos \Theta}{\sigma}}, \quad \sigma \ll 1,
$$

which is obviously suitably normalized to unity when the integral is made over all scattering angles. Using Eq. 3 we find now

$$
w=\frac{1}{2 \pi} \int_{0}^{2 \pi} w_{e} d\left(\phi-\phi^{\prime}\right)=\frac{1}{\sigma} e^{-\frac{1-\mu \mu^{\prime}}{\sigma}} I_{0}\left(\frac{\sqrt{1-\mu^{2}} \sqrt{1-\mu^{\prime 2}}}{\sigma}\right)
$$

where $I_{0}(x)$ is Bessel's modified function of order 0, and use has been made of the formula (Gradshteyn and Ryzhik, 1994, 3.339):

$$
\int_{0}^{\pi} \exp (z \cos x) d x=\pi I_{0}(z) .
$$

In the following we shall use Eq. 47 when computing spectra, taking $\sigma \ll 1$ for the isotropic SPAS limit and $\sigma \gg 1$ for the isotropic large angle scattering regime.

\section{Calculation of the spectrum and angular distribution function}

In this section we outline the procedure to follow in order to calculate the spectral slope and the angular distribution function of the accelerated particles, for an arbitrary choice of the shock velocity and of the scattering properties of the fluid.

For the purpose of making contact with previous literature on the subject, we carry out our calculations with a scattering function $w\left(\mu, \mu^{\prime}\right)$ as given in Eq. 47, choosing a small value of the parameter $\sigma$ for the case of small pitch angle scattering, and a large value of $\sigma$ to reproduce the regime of large angle scattering. Eq. 47 ensures that the scattering remains isotropic in both regimes. However, we wish to stress that we elected to use an isotropic scattering in order to make contact with all other authors (so far), who have only 
used isotropic scattering, even though this is by no means required by our method, which can effectively deal with anisotropic scattering.

It is worth stressing that our method does not require any intrinsic approximations or assumptions on the type of scattering; the only approximations are due to round-off in the numerical solution of the integral equations for $P_{d}$ and $P_{u}$.

The procedure for the calculation of the slope of the spectrum of accelerated particles is as follows: for a given Lorentz factor of the shock $\left(\gamma_{s h}\right)$, the velocity of the upstream fluid $u=\beta_{s h}$ is calculated. We then determine the velocity of the downstream fluid $u_{d}$ from the conventional jump conditions at the shock, after assuming some equation of state for the post-shock fluid. Given $u, u_{d}$ and a scattering function $w$, we can solve the integral equations for $P_{u}$ and $P_{d}$ iteratively. We recall here that these are functions of an entrance and an exit angle, and the convergence of the method can be numerically time consuming, in particular for small values of $\sigma$. Aside from this numerical issue, one should also remember that our equations for $P_{u}$ and $P_{d}$ are analogous to the H-equation of Chandrasekhar (which is however in one dimension only) which is known to admit multiple solutions. It has been shown however (Kelley 1982) that iterative methods always converge to the only physically meaningful solution, although no information is provided on how fast the convergence is achieved.

An important point to keep in mind is that the probability of return from the upstream section is strongly constrained through the condition that all particles must return to the shock, being advected with the fluid. It follows that

$$
\int_{-u}^{1} d \mu^{\prime} P_{u}\left(\mu_{0}, \mu^{\prime}\right)=1
$$

for any value of the entrance angle $\mu_{0}$. It should be stressed however that this integral constraint is not imposed by hand. In other words, though dictated by physical considerations, it is automatically implemented by the integral equation for $P_{u}$ (Eq. 20): it thus provides an important numerical check of our computing accuracy.

The slow convergence of the iterative solution of Eq. 16 has been partly corrected by use of the following ruse. The conditional probability $P_{d}$ obeys the following integral constraint. From Eq. 2 we see that at downstream infinity the condition $\partial f / \partial z=0$ implies that the only physically acceptable solution for the distribution function is $f(\mu)=$ constant $=K$. At downstream infinity we can therefore write

$$
f_{-}(\mu)(u+\mu)=K(u+\mu)=-\int_{-u_{d}}^{1} d \mu^{\prime} f_{+}\left(\mu^{\prime}\right)\left(u+\mu^{\prime}\right) P_{d}\left(\mu^{\prime}, \mu\right)=-\int_{-u_{d}}^{1} d \mu^{\prime} K\left(u+\mu^{\prime}\right) P_{d}\left(\mu^{\prime}, \mu\right),
$$


which implies the condition

$$
\int_{-u_{d}}^{1} d \mu^{\prime}\left(u_{d}+\mu^{\prime}\right) P_{d}\left(\mu^{\prime}, \mu\right)=-\left(u_{d}+\mu\right)
$$

for any value of the exit angle $\mu$. Since the function $P_{d}$ depends only on the scattering properties of the fluid, which are assumed to be independent of $z$, Eq. 50 also applies to any $z$, including $z=0$ which is the position of the shock.

This condition is of course automatically satisfied at the end of our calculations, while it is only approximately satisfied during the iterative solution of Eq. 16. We have found out that, by correcting the normalization of $P_{d}$ so that it obeys the previous integral constraint to hold at the end of every iteration, the convergence is much accelerated, above all in the SPAS regime. We are unable to explain why this occurs, but shortening of the computation times by a factor of 3, or more, have thus been achieved.

Once the two functions $P_{u}$ and $P_{d}$ have been calculated, the slope of the spectrum, as discussed in paper I, is given by the solution of the equation:

$$
\left(u_{d}+\mu\right) g(\mu)=\int_{-u_{d}}^{1} d \xi Q^{T}(\xi, \mu)\left(u_{d}+\xi\right) g(\xi),
$$

where

$$
Q^{T}(\xi, \mu)=\int_{-1}^{-u_{d}} d \nu P_{u}(\nu, \mu) P_{d}(\xi, \nu)\left(\frac{1-u_{r e l} \mu}{1-u_{r e l} \nu}\right)^{3-s} .
$$

Here $u_{\text {rel }}=\frac{u-u_{d}}{1-u u_{d}}$ is the relative velocity between the upstream and downstream fluids and $g(\mu)$ is the angular part of the distribution function of the accelerated particles, which contains all the information about the anisotropy. A point to notice concerns the function $P_{u}$ in Eq. (52): all variables and functions here are evaluated in the downstream frame, while the $P_{u}$ calculated though Eq. (20) is in the frame comoving with the (upstream) fluid. The $P_{u}$ that need to be used in Eq. (52) is therefore

$$
P_{u}(\nu, \mu)=P_{u}(\tilde{\nu}, \tilde{\mu}) \frac{d \tilde{\mu}}{d \mu}=P_{u}(\tilde{\nu}, \tilde{\mu})\left[\frac{1-u_{r e l}^{2}}{\left(1-u_{r e l} \mu\right)^{2}}\right] .
$$

The solution for the slope $s$ of the spectrum is found by solving Eq. 51. In general, this equation has no solution but for one value ${ }^{2}$ of $s$. Finding this value provides not only the slope of the spectrum but also the angular distribution function $g(\mu)$.

\footnotetext{
${ }^{2}$ The fact that the solution is unique could not be demonstrated rigorously, but in our calculations we never found cases of multiple solutions.
} 


\section{Comparison with previous results}

In this section we apply the method detailed above to several cases that have already been discussed in the literature, in order to show that the approach can be successfully applied to a variety of cases. More specifically, we consider the following test situations:

- Since our method is not based on the assumption of small pitch angle scattering, we can demonstrate that the slope of the spectrum of particles accelerated at Newtonian shocks is independent of the scattering function $w\left(\mu, \mu^{\prime}\right)$, although the functions $P_{d}$ and $P_{u}$ in general do depend on the choice of $w$.

- To our knowledge, the only calculation of the functions $P_{d}$ and $P_{u}$ which applies to shocks of arbitrary velocity for the case of large angle scattering is that of Kato \& Takahara (2001). We compare our results for $P_{d}$ and $P_{u}$ with those presented in that paper for a scattering function which reproduces the physical case of large angle scattering.

- The slope of the spectrum of accelerated particles in the relativistic regime for the case of large angle scattering was compared with the results of Monte Carlo calculations carried out by Ellison, Jones and Reynolds (1990).

- The case of small pitch angle scattering was assumed in most previous calculations. We test our approach in this regime in two ways: 1) by showing that the slope of the spectrum of accelerated particles in the SPAS approximation, as computed through our method for several values of the shock Lorentz factor, compares well with the results presented in (Kirk et al., 2000); 2) by comparing our angular distribution function with that obtained by Kirk \& Schneider (1987) for a given relativistic shock velocity.

\subsection{The Newtonian case}

The theoretical approach presented here allows us to determine the spectrum and the anisotropy in the particle distribution for arbitrary shock velocity and arbitrary scattering properties of the fluid. In this section we show that we can reproduce the results expected in the non-relativistic limit. We calculated the spectral slope and the angular part of the distribution function for several values of the shock velocity and shock compression factor $r$. We also calculated the return probability from the downstream and upstream sections. The expected return probability in the Newtonian limit is $P_{r e t}^{d}=1-4 u_{d}$, very accurately reproduced by our calculation, for instance for $u_{d}=10^{-2}\left(P_{\text {ret }}^{d}=0.96\right)$ and for $u_{d}=2 \times 10^{-2}$ 

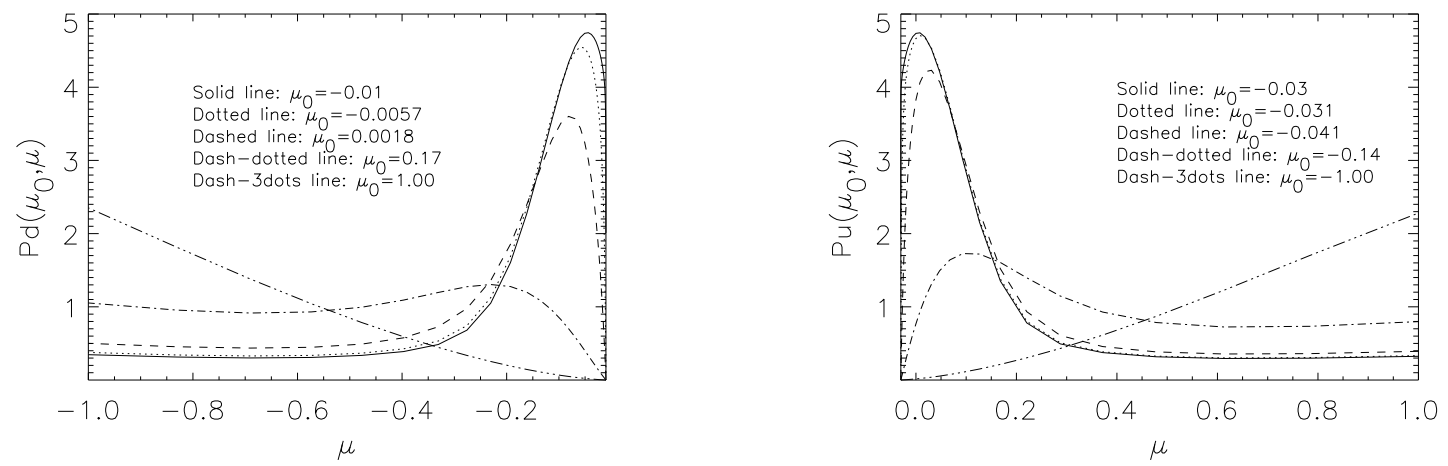

Fig. 1. - Probability functions $P_{d}\left(\mu_{0}, \mu\right)$ and $P_{u}\left(\mu_{0}, \mu\right)$ for $u=0.03$ and $u_{d}=0.01$ for $\sigma=0.01$, at fixed values of $\mu_{0}$ (as indicated).

$\left(P_{\text {ret }}^{d}=0.92\right)$. In all cases that we considered, the condition

$$
\int_{-u}^{1} d \mu^{\prime} P_{u}\left(\mu_{0}, \mu^{\prime}\right)=1
$$

is confirmed for any value of $\mu_{0}$ within a part in $10^{4}$. As a matter of fact, we checked that the above equality is satisfied in all cases we studied, relativistic or otherwise.

The slope of the spectrum of accelerated particles for $u=3 \times 10^{-2}$ and $u_{d}=10^{-2}$ (compression factor 3) as calculated with our approach is 4.5, in agreement with the theoretical prediction (Bell 1978). We obtain the same slope in both cases of small pitch angle scattering with $\sigma=0.01$ and large angle scattering, that we simulate by choosing $\sigma=100$ (but any large value gives the same result). The fact that in the newtonian limit the slope of the spectrum does not depend on the scattering properties of the fluid is also in agreement with the theoretical prediction of Bell (1978). It is worth stressing that this universality in the spectrum of the accelerated particles does not reflect in the universality of the return probabilities $P_{d}$ and $P_{u}$. In Fig. 1 we plot $P_{d}\left(\mu_{0}, \mu\right)$ and $P_{u}\left(\mu_{0}, \mu\right)$ as functions of $\mu$ for the values of $\mu_{0}$ indicated in the figure and assuming $u=0.03, u_{d}=0.01$ and $\sigma=0.01$. The same functions are plotted in Fig. 2 for the case of large angle scattering $(\sigma=100)$.

\subsection{The probability functions $P_{d}$ and $P_{u}$ for the relativistic regime and large angle scattering}

As stressed above, our method is based on the introduction of the two probability distributions $P_{d}$ and $P_{u}$, that can be calculated by solving two integral equations, Eqs. (16) 

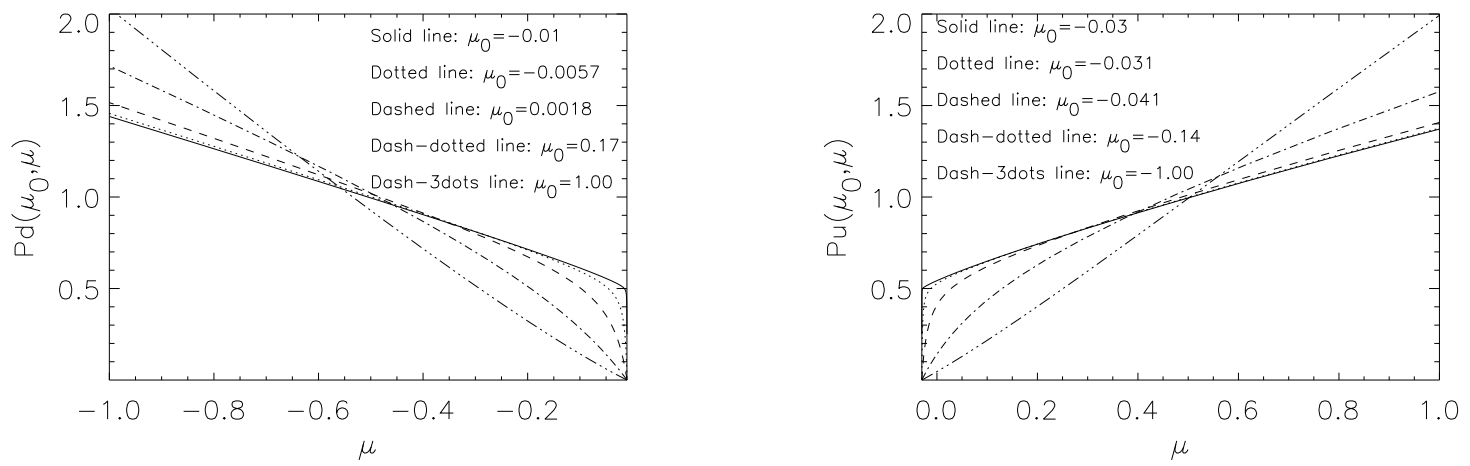

Fig. 2.- Probability functions $P_{d}\left(\mu_{0}, \mu\right)$ and $P_{u}\left(\mu_{0}, \mu\right)$ for $u=0.03$ and $u_{d}=0.01$ for $\sigma=100$ (large angle scattering), at fixed values of $\mu_{0}$ (as indicated).
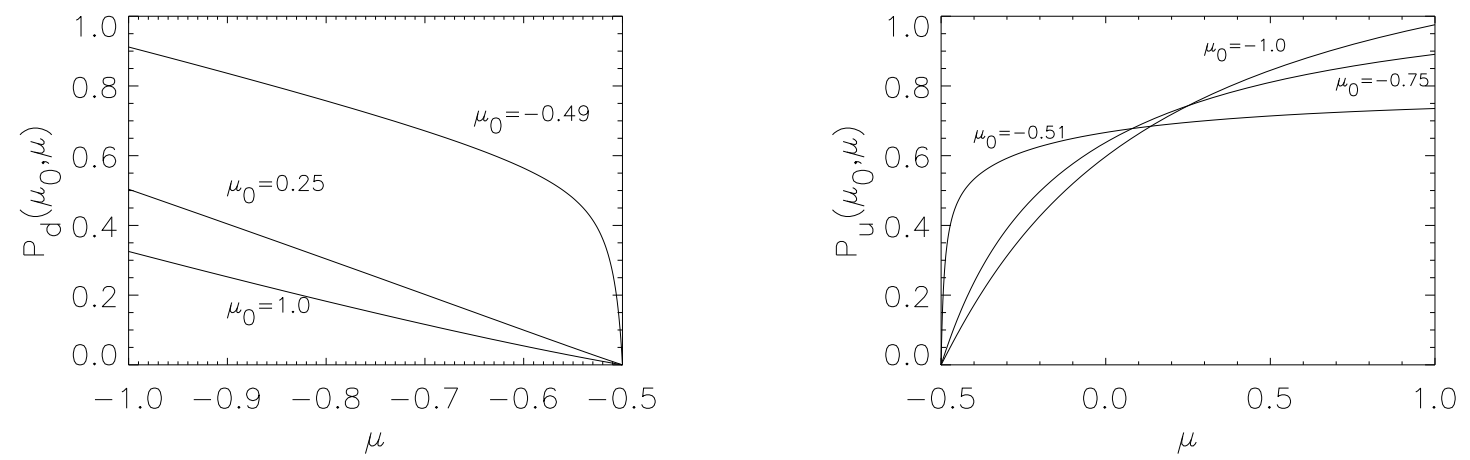

Fig. 3.- Return probabilities $P_{d}\left(\mu_{0}, \mu\right)$ (left) and $P_{u}\left(\mu_{0}, \mu\right)$ (right) that a particle entering the downstream (upstream) region with an angle having cosine $\mu_{0}$, exits it with an angle having cosine $\mu$, for the values of $\mu_{0}$ as indicated.

and (20). The only other approach that we are aware of, that introduces these two functions is that presented by Kato \& Takahara (2001), based on the theory of random walk. We apply our calculations to reproduce Fig. 6 of their paper, obtained for a fluid speed $u=0.5$ both upstream and downstream and in the regime of large angle scattering.

We plot our results in Fig. 3 for the functions $P_{d}$ (left panel) and $P_{u}$ (right panel). We carry out our calculations for exactly the same values of $\mu_{0}$ as indicated in Fig. 6 of Kato \& Takahara (2001) and reported in our Fig. 3 as well. It is worths stressing that for the downstream section of the fluid $u_{d}+\mu_{0}>0$ and $u_{d}+\mu<0$, while for the upstream section these relations have opposite signs. 
There is perfect agreement between our results and those of Fig. 6 of Kato and Takahara (2001), which is further evidence that our method is perfectly able to reproduce previous results, despite the absolute absence of any physical approximation.

\subsection{The spectrum for the case of large angle scattering}

The spectrum of the accelerated particles for the case of pure large angle scattering was calculated by Kato \& Takahara (2001) and there compared with the previous Monte Carlo result of Ellison et al. (1990). The case of mixed small and large angle scattering was investigated by Kirk \& Schneider (1988).

We apply our method to determine the spectrum of accelerated particles for pure large angle scattering and compare our results with those previously obtained by Kato \& Takahara (2001) and by Ellison et al. (1990), which were slightly discrepant for relativistic shock speeds. The spectral slopes calculated according with the procedure defined in the previous section are plotted in Fig. 4 for shock speeds $u=0.8$ and $u=0.9$ as functions of the compression factor $r$ at the shock. The crosses and diamonds are the results of our calculations, while the continuous curves represent the interpolation provided by Ellison et al. (1990) to their Monte Carlo results.

It is remarkable that, while the probability distributions $P_{d}$ and $P_{u}$ computed by us and by Kato and Takahara (2001) agree perfectly, our slopes differ from theirs. We speculate that this is due to the different method of computation. Kato and Takahara (2001) use Peacock's recipe:

$$
P<G>^{s-3}=1
$$

where $\langle G\rangle$ is the average energy fractional gain when a whole cycle upstream $\rightarrow$ downstream $\rightarrow$ upstream is performed. However, in paper I, we showed that the correct formula is

$$
P<G^{s-3}>=1
$$

which obviously differs from the previous one. The fact that our computations, on the one hand, match the functions $P_{d}$ and $P_{u}$ of Kato and Takahara, but on the other one reproduce so perfectly the slopes of Ellison, Jones and Reynolds (1990) (which use neither our approach nor Peacock's) supports our claim that our formula is the correct one. 


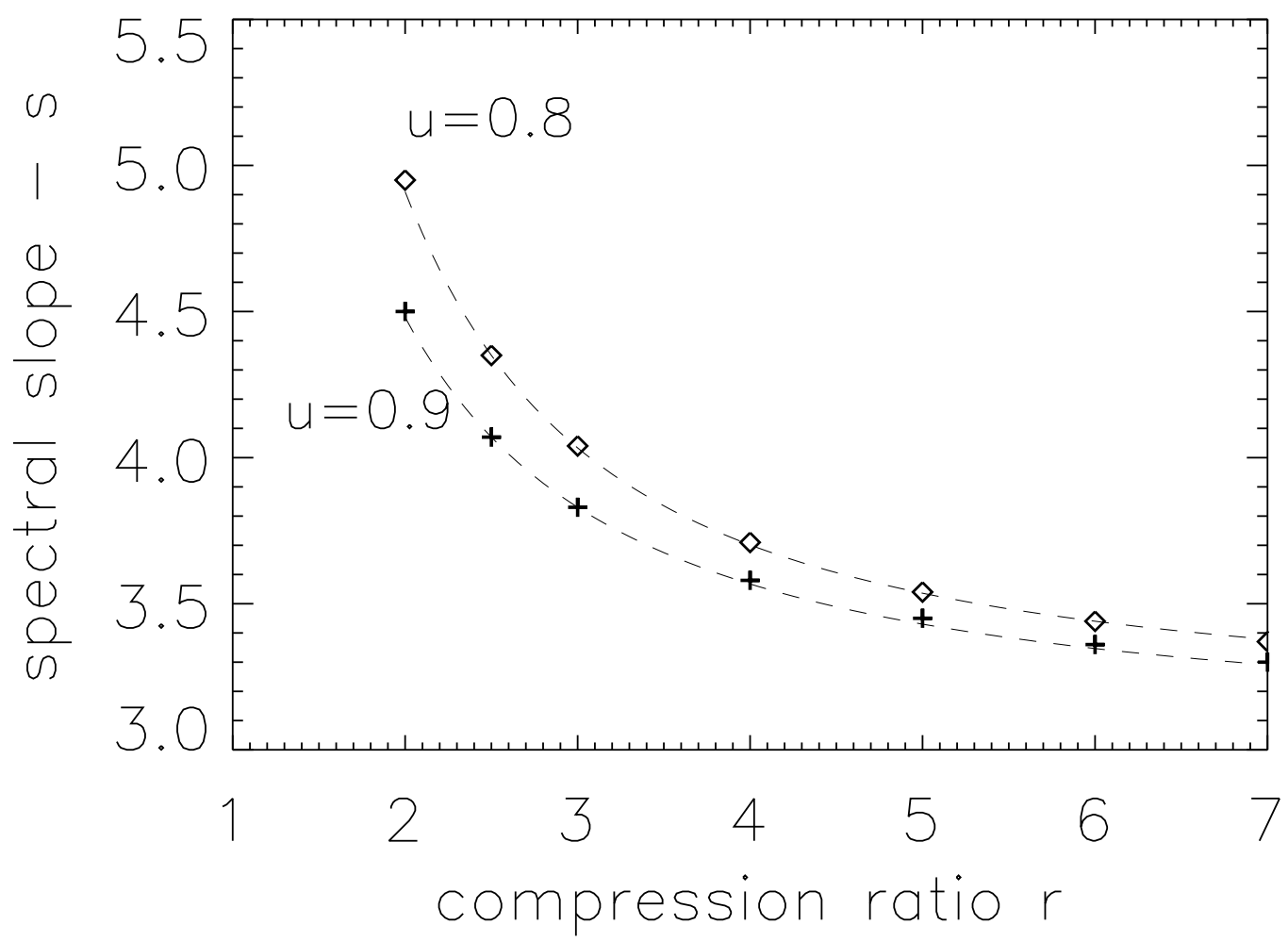

Fig. 4. - Slope of the spectrum of accelerated particles for a shock with speed $u=0.8$ (diamonds - upper curve) and $u=0.9$ (crosses - lower curve) in the regime of large angle scattering, as a function of the compression ratio. The continuous lines are the fit to the results of Ellison et al. (1990).

\subsection{The SPAS limit}

Most calculations previously presented in the literature adopt the assumption of SPAS, in order to reduce the transport equation (Eq. 2) to a Fokker-Planck equation, as shown in section 3. In the approach presented in Paper I and here, the scattering occurs with arbitrary characteristics, embedded in the scattering function $w\left(\mu, \mu^{\prime}\right)$. The case of a $w$ independent of pitch angles is that of strong scattering, which is expected to be achieved for large amplitude turbulence. The case of small pitch angle scattering in the assumption of isotropic scattering can be modeled in our approach by taking a peaked shape for the scattering function, as explained in section 4 , with $\sigma \ll 1$. 
We expect that in the limit of SPAS the result should not depend upon the detailed form of the scattering function, provided this is strongly peaked (see Section 3).

For a given Lorentz factor of the shock $\left(\gamma_{s h}\right)$, the velocity of the upstream fluid $u=\beta_{s h}$ is calculated. For the purpose of comparing our results with those of Kirk et al. (2000), we adopt a Synge (1957) equation of state for the downstream fluid and assume that the magnetic field is not dynamically important. These are the same assumptions as in (Kirk et al. 2000).

We can therefore calculate the velocity of the downstream fluid $u_{d}$ from the conservation of mass, momentum and energy at the shock surface. The values of $u$ and $u_{d}$ are given in Table 1. Given these velocities and a width $\sigma$ for the scattering function, we can solve the integral equations for $P_{u}$ and $P_{d}$ iteratively.

\begin{tabular}{|c|c|c|c|}
\hline$\gamma_{s h} \beta_{s h}$ & $u$ & $u_{d}$ & slope \\
\hline 0.04 & 0.04 & 0.01 & 4.00 \\
0.2 & 0.196 & 0.049 & 3.99 \\
0.4 & 0.371 & 0.094 & 3.99 \\
0.6 & 0.51 & 0.132 & 3.98 \\
1.0 & 0.707 & 1.191 & 4.00 \\
2.0 & 0.894 & 0.263 & 4.07 \\
4.0 & 0.97 & 0.305 & 4.12 \\
5.0 & 0.98 & 0.311 & 4.13 \\
\hline
\end{tabular}

Following the procedure outlined in section 4, we determine the slope of the power law spectrum and the angular part $g(\mu)$ of the distribution function.

The slopes predicted by our calculations for different values of the shock velocity are shown in Table 1 for $\sigma=0.01$ and agree well with those of Kirk et al. (2000). In Fig. 5 we also plot the distribution functions obtained for all the cases reported in Table 1 (the upper panel refers to the first four lines in Table 1, while the lower panel refers to the relativistic regime, namely the last four lines in Table 1).

This figure shows the expected phenomenon of increasing anisotropy in the distribution funtion when the shock speed increases: for non-relativistic speeds the distribution function is almost perfectly independent of the pitch angle $\mu$, but it becomes more and more anisotropic in the trans-relativistic regime and in the fully relativistic regime.

A necessary condition for the SPAS regime to be at work is that $\sigma \ll 1 / 4 \gamma_{s h}^{2}$. This implies that the SPAS assumption requires increasingly lower values of $\sigma$ when the Lorentz 

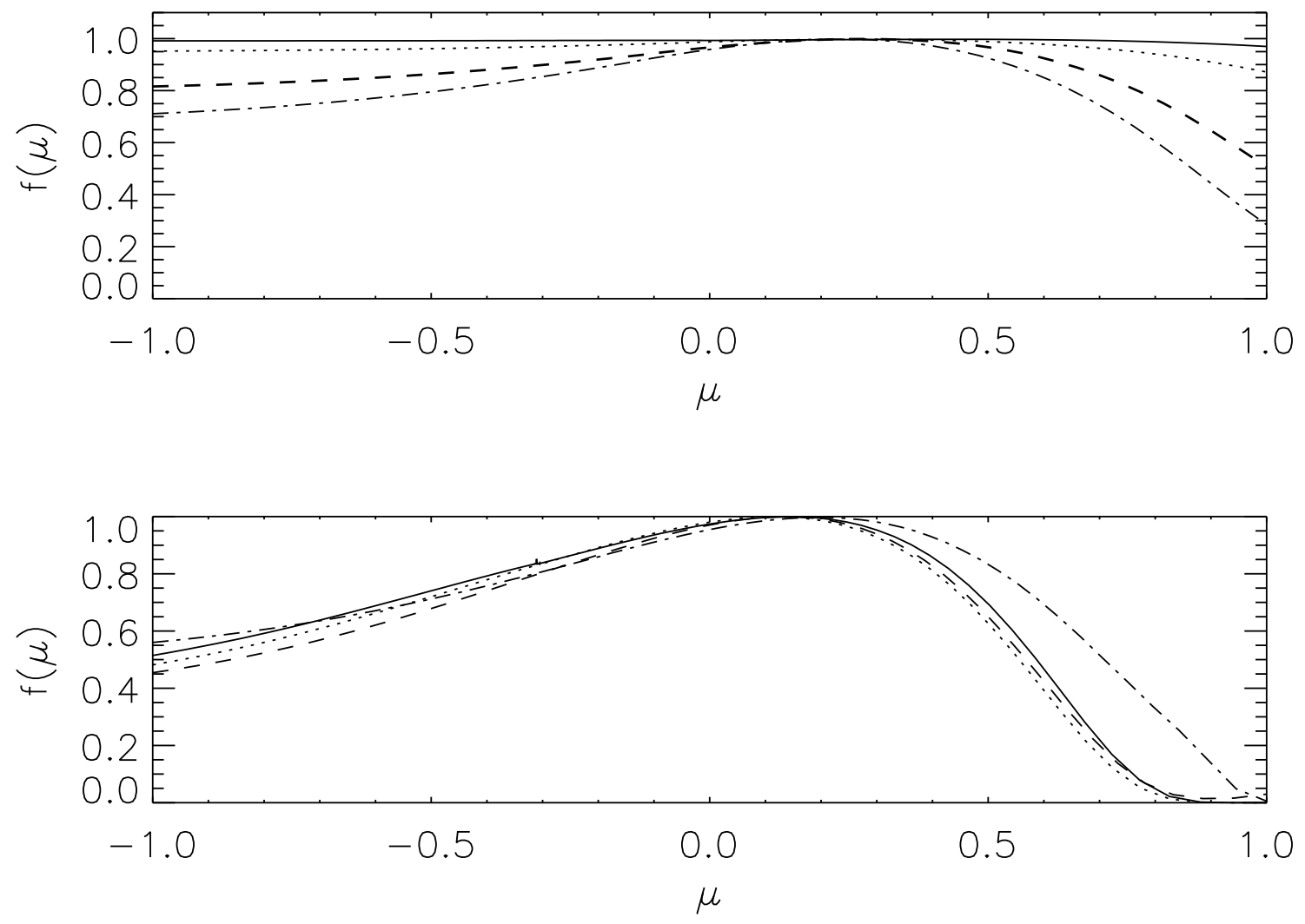

Fig. 5.- Upper panel: DF for $\gamma_{s h} \beta_{s h}=0.04$ (solid line), $\gamma_{s h} \beta_{s h}=0.2$ (dotted line), $\gamma_{s h} \beta_{s h}=0.4$ (dashed line) and $\gamma_{s h} \beta_{s h}=0.6$ (dash-dotted line). Lower panel: DF for $\gamma_{s h} \beta_{s h}=1$ (dash-dotted line), $\gamma_{s h} \beta_{s h}=2$ (dashed line), $\gamma_{s h} \beta_{s h}=4$ (dotted line) and $\gamma_{s h} \beta_{s h}=5$ (solid line).

factor of the shock increases. For $\sigma=0.01$, the maximum Lorentz factor for which we can assume to be in the SPAS regime is $\gamma_{s h} \sim 5$, and in fact we can already see that the slopes that we obtain for $\gamma_{s h} \beta_{s h}=5$ are slightly smaller than those of Kirk et al. (2000). This effect will be discussed in detail in a forthcoming paper.

It is worth reminding that the SPAS approximation is in fact expected to be broken in Nature in the relativistic regime: this is due to the fact that a FP equation describes well the scattering only when particles scatter many times within a given angle. In the upstream section, particles are caught up by the shock as soon as their deflection angle with respect to the normal to the shock is larger than $1 / \gamma_{s h}$. For relativistic shocks, this quantity becomes small and eventually comparable with the average deflection angle per unit length, and a 
description by means of a Fokker-Planck equation becomes inadequate, as remarked many times in the literature (Kirk et al., 2000). We stress here that the fact that our method is not based upon the SPAS approximation, but on the more general Eq. 2, makes it more widely applicable then previous approaches. Its potential for a more correct investigation of the large $\gamma$ limit will have to be investigated elsewhere.

An additional evidence of how important these effects can be for the determination of the correct distribution function of the accelerated particles is illustrated below. We calculate here the spectrum and the function $g(\mu)$ for $u=0.9$ and for the relativistic equation of state for the downstream gas $\left(u u_{d}=1 / 3\right)$, as adopted by Kirk \& Schneider (1987). We carry out our calculations for $\sigma=0.05,0.03,0.01$ and 0.005 . The slopes in the four cases are very close to each other $(s=4.68,4.69,4.71,4.71)$ but the angular distribution functions (normalized to be unity at the peak) appear slightly different. It is remarkable however that when the SPAS regime is approached, the DF converges exactly to that found by Kirk \& Schneider (1987), as illustrated in Fig. 6, where we plotted $g(\mu)$ for the four cases mentioned above: compare our results with Kirk and Schneider's Fig. 6. The slope that we obtain is also in perfect agreement with Kirk \& Schneider (1987).

\section{Conclusions}

In Paper I, a new analytic approach to the calculation of the spectrum of particles accelerated at a shock surface moving with arbitrary velocity was proposed. In the present paper we described the procedure to calculate the probability functions $P_{d}\left(\mu_{0}, \mu\right)$ and $P_{u}\left(\mu_{0}, \mu\right)$ introduced in Paper I. These two functions allow us to calculate the spectrum and the angular distribution function of the accelerated particles at the shock, for arbitrary shock velocity and for arbitrary scattering function $w$.

We tested the approach in several situations, in order to show that all results previously appeared in the literature could be successfully reproduced. In particular the following tests have been performed:

- we confirmed the universality of the slope of the power law spectrum of accelerated particles in the case of Newtonian shocks. More specifically we proved that such slope is roughly independent of the scattering properties of the medium, as described by the scattering function $w$. It should be stressed that such universality does not extend to the probability functions $P_{d}$ and $P_{u}$, which are instead strongly dependent upon the scattering properties. Despite the difference between these probability functions, their combination results in an angular part of the distribution function of accelerated 


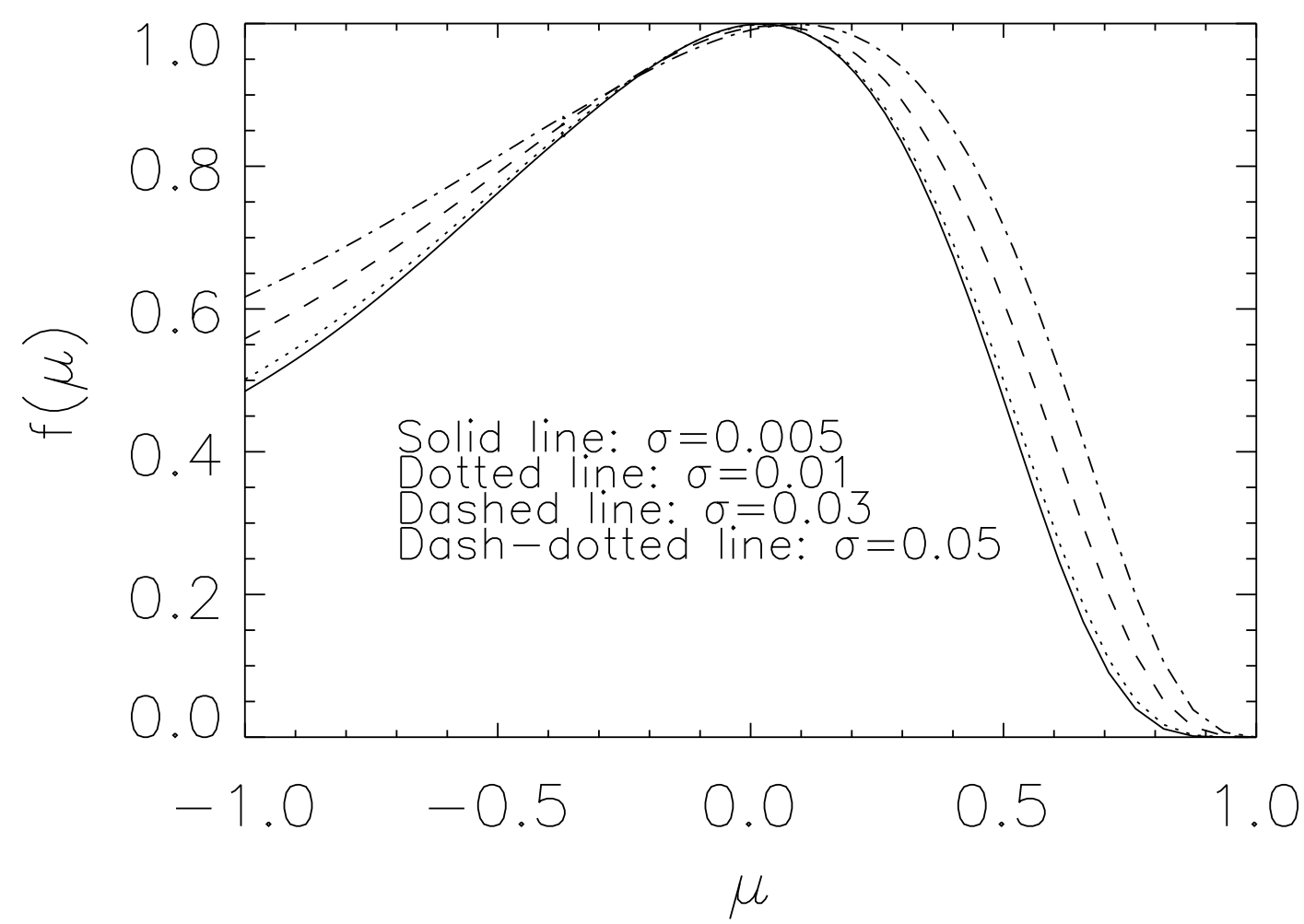

Fig. 6. - The angular distribution function $g(\mu)$ for $u=0.9$ and for the relativistic equation of state for the downstream gas $u u_{d}=1 / 3$. The solid, dotted, dashed and dot-dashed lines refer respectively to $\sigma=0.005,0.01,0.03$ and 0.05 .

particles which is approximately flat, as expected in the Newtonian limit (isotropy).

- We checked the correctness of the probability functions $P_{d}$ and $P_{u}$ in the case of large angle scattering by comparing our results with those of Kato \& Takahara (2001). We emphasize that these two functions contain all the physical information about the spectrum and angular distribution of the accelerated particles.

- We reproduce exceptionally well the results of the Monte Carlo experiments of Ellison et al. (1990) on the slope of the spectrum of accelerated particles as a function of the shock compression ratio for different shock speeds, in the large angle scattering regime, but not Kato and Takahara's (2001) results. We ascribed this discrepancy to Kato and Takahara's use of the (incorrect) Peacock's formula for the spectral slope. 
- We carried out calculations for the case of a scattering function very peaked as a function of the difference between the cosines of the entrance and exit angles. We adopted a $w$ in the form given by our Eq. 47. The case of small $\sigma$ is expected to describe the limit of isotropic small pitch angle scattering, which is adopted in most literature on shock acceleration. We showed that in this limit the transport equation (Eq. 2) reduces to a Fokker-Planck equation, identical to that used for instance by Kirk \& Schneider (1987) and Kirk et al. (2000). We carried out computations for $\sigma=0.05-0.005$, and we compared our predicted spectral slopes with those of Kirk et al. (2000) for different values of the shock velocity. Our results are illustrated in Table 1 and show that our method reproduces faily well the SPAS results. Moreover, our approach shows the expected deviations from the SPAS regime when the condition $\sigma \ll 1 /\left(4 \gamma_{s h}^{2}\right)$ is violated.

- We have also compared our distribution function at the shock with that published by Kirk and Schneider (1987), obtaining very good agreement, and an identical slope. We also show that the distribution function becomes increasingly different when the strict SPAS regime is broken (Fig. 6).

On the basis of the tests carried out to check our approach, we can claim that this method of calculation of the spectrum and angular distribution function of particles accelerated at shocks of arbitrary velocity and arbitrary (but spatially homogeneous) scattering properties of the fluid is fully successful in reproducing old results, and it can now be applied to situations which go beyond the well studied case of small pitch angle scattering. In a forthcoming paper we will describe the potential of this new approach to describe particle acceleration at ultra-relativistic shocks, including the effect of a possible large scale magnetic field.

A sincere thanks is due to the referee, whose selfless work has considerably improved an early version of this work.

\section{REFERENCES}

Bell, A.R., 1978, MNRAS, 182, 147; Bell, A.R., 1978, MNRAS, 182, 443.

Chandrasekhar, S., 1949, Radiative Transfer, Dover, New York.

Ellison, D., Jones, F.C., and Reynolds, S.P., 1990, ApJ, 360, 702.

Freiling, G., Vietri, M., and Yurko, V., 2003, Letters in Mathematical Physics, 64, 65.

Gleeson, L.J., Axford, W.I., 1967, ApJL, 149, L115. 
Gradshteyn, I.S., Ryzhik, I.M., 1994, Table of Integrals, Series and Products, Academic Press, S.Diego.

Kato, T.N., and Takahara, F., 2001, MNRAS, 321, 642.

Kelley, C.T., 1982, J. Math. Phys., 23, 2097.

Kirk, J.G., Guthmann, A.W., Gallant, Y.A., and Achterberg, A., 2000, ApJ, 542, 235.

Kirk, J.G., and Schneider, P., 1987, Ap.J., 315, 425.

Kirk, J.G., and Schneider, P., 1988, A \& A, 201, 177.

Landau, L.D., Lifshitz, I.M., 1984, Fisica Cinetica, Editori Riuniti, Roma.

Peakock, J.A., 1981, MNRAS, 196, 135.

Synge, J.L., 1957, The relativistic gas (North-Holland, Amsterdam)

Vietri, M., 2003, ApJ, 591, 954. 